\title{
EL IMPACTO DEL CORONAVIRUS EN LA FIGURA PRESIDENCIAL ARGENTINA
}

\section{The Impact of the Coronavirus on Argentine Presidential Standing O Impacto do Coronavírus na Figura Presidencial Argentina}

\author{
LUCAS KLOBOVS @ Iklobovs@poliarquia.com ${ }^{1}$ \\ ${ }^{1}$ Poliarquía Consultores, Universidad Nacional de Tres de Febrero \\ Envío: 2020-06-23 \\ Aceptado: 2020-11-19 \\ First View: 2020-12-18 \\ Publicación: 2021-11-08
}

\begin{abstract}
Palabras clave:
Resumen

Coronavirus;

Aprobación

Alberto Fernández asumió la presidencia en diciembre de 2019 y el debate so-

presidencial;

Liderazgo;

Gestión;

Autoridad bre si su liderazgo se encontraba eclipsado por la figura de la vicepresidenta Cristina Kirchner estaba instalado. En este contexto, el coronavirus estalla en Argentina y el presidente logra altos niveles de aprobación (incluso por encima de los que registró luego de su asunción) a medida que la preocupación social por la pandemia aumentaba. ¿Cuál es el impacto real del coronavirus sobre la figura del presidente? A su vez, este salto en la valoración de su gestión impulsa la consolidación de su autoridad, pero, al mismo tiempo, abre el interrogante sobre si se trata de una consolidación real o momentánea.
\end{abstract}

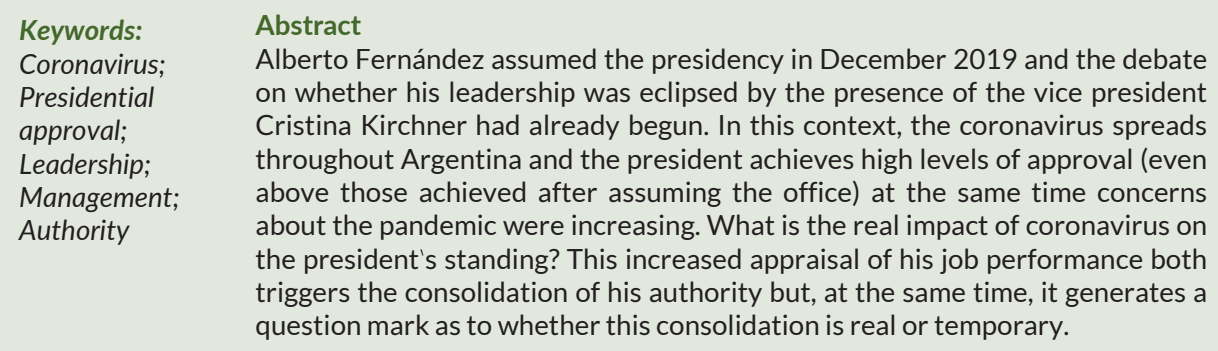


Palavras-chave: Coronavírus; Aprovação presidencial; Liderança; Gestão; Autoridade
Resumo

Alberto Fernández assumiu a presidência em dezembro de 2019 e o debate sobre se sua liderança foi ofuscada pela figura da vice-presidente Cristina Kirchner foi instalada. Nesse contexto, o coronavírus irrompe na Argentina e o presidente atinge níveis elevados de aprovação (até acima dos que registrou após sua posse) à medida que aumenta a preocupação social com a pandemia. Qual é o real impacto do coronavírus na figura do presidente? Por sua vez, este salto na avaliação da sua gestão impulsiona a consolidação da sua autoridade mas, ao mesmo tempo, abre a questão se se trata de uma consolidação real ou temporária.

\section{INTRODUCCIÓN}

En 2019, previo al inicio de la campaña electoral presidencial en Argentina, se produjo un hecho inédito para la historia política del país. La exmandataria Cristina Kirchner ungió como candidato a presidente a Alberto Fernández, secundada por ella misma: por primera vez, un candidato a vicepresidente elegía quién encabezaría la fórmula.

Desde aquel momento se ha debatido sobre el rol y el poder que tendría la vicepresidenta en el gobierno del Frente de Todos. La condición de ser candidato a presidente a partir de la decisión del otro componente de la fórmula ubicó al actual mandatario en una posición de presunta debilidad política. El actual mandatario era un dirigente con trayectoria política, pero no era un actor relevante en la agenda de la opinión pública. Incluso, hasta ese momento, ningún analista lo consideraba, siquiera, como posible candidato y, menos, como potencial presidente $^{1}$. En otras palabras, llegó al Ejecutivo Nacional, en gran parte, gracias al impulso y aporte electoral de Cristina Kirchner. Por sí solo difícilmente hubiese llegado.

Y aquí radicaba su principal desafío político en la construcción de poder. Maquiavelo ya lo analizó en el Príncipe. El florentino distinguía los tipos de principados. Uno de ellos, los que fueron adquiridos con fuerza ajena (no por méritos o poder propio) son los más difíciles de gobernar porque el nuevo príncipe depende de aquella fuerza ajena. Por lo tanto, para evitar ser derrocado, Maquiavelo recomendaba, en esos casos, eliminar el linaje del príncipe anterior. Así se evitaría que este o su familia recuperaran el poder.

Este consejo lo comprendió muy bien el expresidente Néstor Kirchner cuando, luego de llegar al poder por fuerza ajena (el aparato y el apoyo del entonces

1. Más allá de su amplia trayectoria como dirigente y militante político, una expresión de su menor relevancia pública en términos electorales es que en enero de 2013 fue la última vez que se midió su valoración pública en una encuesta de Poliarquía. 
presidente Eduardo Duhalde) se alejó de él en las elecciones legislativas de 2005. Por aquel entonces el kirchnerismo y el duhaldismo debatían la conformación de las listas de candidatos a diputados y senadores nacionales. Finalmente, no hubo acuerdo y ambos espacios se presentaron por separado. Por un lado, Hilda Chiche Duhalde y, por el otro, Cristina Kirchner. Ambas encabezaron la lista de senadores nacionales por la provincia de Buenos Aires. El rotundo triunfo del kirchnerismo representó la confirmación del liderazgo de los Kirchner en la política argentina.

Casi quince años después, los consejos de Maquiavelo retoman su protagonismo. Alberto Fernández llegó al Ejecutivo Nacional impulsado por Cristina Kirchner y su desafío era construir poder simbólico y consolidar su autoridad y liderazgo.

La gran diferencia con aquel episodio de 2005 es que, en este caso, quien fue la artífice principal en la conformación de la fórmula se encuentra dentro del mismo gobierno, lo cual dificultaba aún más el cometido. Lo que sí quedaba claro es que el presidente tenía como principal desafío político la construcción de poder, liderazgo y autoridad. Como dice Martín Rodríguez Yerba "es irrelevante entrar en el juego cansador de si gobierna ella o gobierna él. La existencia de la duda es el triunfo de Cristina. Y la mayor amenaza a la autoridad del Presidente" (Rodríguez Yerba, 2020).

Este debate no era un tema exclusivo de los periodistas, académicos y analistas: la población argentina tenía dudas sobre quién era el responsable de la toma de decisiones en el gobierno. Según una encuesta nacional realizada por Poliarquía Consultores en el mes de diciembre de 2019 (mes de su asunción), solamente un $14 \%$ señalaba que Alberto Fernández sería quien tuviera el poder y Cristina Kirchner acataría sus decisiones. Es más, casi el doble (26\%) creía que la vicepresidenta sería quien tuviera el poder y Alberto Fernández acataría sus decisiones. El 32 \% creía que el presidente compartiría el espacio con Cristina Kirchner en una convivencia pacífica y, por último, el $22 \%$ consideraba que se desataría una lucha de poder entre Alberto Fernández y Cristina Kirchner. 
Tabla 1. Según su opinión, ¿cuál de las siguientes opciones refleja mejor la relación que tendrán Alberto Fernández y Cristina Kirchner?

$\%$

Alberto Fernández será quien tenga el poder y Cristina Kirchner acatará sus decisiones

Alberto Fernández y Cristina Kirchner compartirán el poder en una convivencia pacífica

Se desatará una lucha de poder entre Alberto Fernández y

Cristina Kirchner

Cristina Kirchner será quien tenga el poder y Alberto Fernández acatará sus decisiones

NS/NC

Total
14 161

26 205

6

100

970

Encuesta telefónica (sistema CATI) a mayores de 18 años que residen en ciudades de más de 10000 habitantes, en hogares con teléfono fijo. El campo se realizó en diciembre de 2019.

Fuente: Poliarquía Consultores.

En este contexto de presunta debilidad política, la construcción del liderazgo presidencial debía ser uno de sus objetivos. A comienzos del año se especulaba que una reestructuración exitosa de la deuda y un posterior inicio de un proceso de reactivación económica y crecimiento serían la piedra angular para la consolidación del liderazgo de Alberto Fernández. Pero mientras no se conocían avances en la negociación de la deuda y la economía argentina seguía sumergida en un letargo, explotaba el coronavirus en el mundo y en el país, complicando aún más la economía global y local. A pesar de que la pandemia evaporaba la carta que podría haber usado el presidente, el coronavirus le daba, al mismo tiempo, una nueva oportunidad: consolidar su autoridad y liderazgo en una época de incertidumbre, crisis y medidas de aislamiento social.

La consolidación del liderazgo de un presidente tiene un factor fundamental: el apoyo de la ciudadanía. Neustadt (1990) señala que la fortaleza o la debilidad de un presidente dependen de su capacidad para influir en el comportamiento de los funcionarios de su gobierno. Y este poder de convencimiento se sustenta en su reputación profesional y en su prestigio público. En cuanto a su reputación, Alberto Fernández es un dirigente con amplia trayectoria política, ha ocupado cargos en los gobiernos desde el retorno de la democracia en Argentina hasta llegar a ser nombrado por Néstor Kirchner como jefe de Gabinete, siendo la persona que más ha 
perdurado en dicho cargo en la historia política del país. Por su parte, el prestigio se construye a partir de la opinión de los ciudadanos sobre el gobierno del presidente (citado por Rivas Otero, 2012, p. 327): mejor valoración de su gestión, mayor prestigio y mayor fortaleza. En otras palabras, analizar la popularidad de un presidente es la puerta para comprender su poder (citado por Paul Gronke y Brian Newman, 2015, p. 501).

A su vez, Pedro Magalhães (2020) resalta la importancia de estudiar la aprobación presidencial basándose en dos aspectos. En primer lugar, es la opinión pública la que determina quién gobierna y por cuánto tiempo. Y en segundo lugar, las percepciones de la sociedad condicionan lo que los gobiernos ejecutan y las medidas que toman.

Por último, Newman y Forcehimes (2010) afirman que el estudio de la aprobación presidencial ocupa un rol protagónico en la política norteamericana ya que se trata de un indicador del capital político del presidente.

Fundamentada en estos marcos conceptuales, esta investigación aborda la evaluación de la gestión de Alberto Fernández que realizan los argentinos como elemento central para la consolidación de su liderazgo y autoridad luego del primer contagio por COVID-19 en el país.

\section{TEORÍAS EXPLICATIVAS DE LA APROBACIÓN PRESIDENCIAL}

La mayoría de los estudios sobre los condicionantes de la aprobación presidencial coinciden en que existen cinco motivos que impulsan una valoración positiva hacia la administración gobernante.

En primer lugar, el efecto "luna de miel", por el cual luego de las elecciones el presidente tiene altos niveles de popularidad, los cuales se van deteriorando con el devenir de la gestión para recuperarse, en parte, cerca de la finalización de su mandato (Carlin, Hartlyn, Hellwig, Love, Martínez-Gallardo y Singer, 2018).

Enmarcado en esta corriente, Stimson (1976) argumentó que el deterioro de la popularidad presidencial luego de los primeros meses de gobierno es el resultado de expectativas sobreestimadas de la sociedad, las cuales son consecuencia de una exitosa campaña electoral. La recuperación hacia el final del mandato era, nuevamente, una acumulación de expectativas a medida que comienza la campaña de reelección (citado por Gronke y Newman, 2003, p. 503). En cambio, Brody (1991) observó que los períodos de luna de miel son consecuencia de una cobertura positiva de los medios al comienzo de un período: la mayoría de los líderes de opinión tienden a ofrecer pocas críticas, reteniendo el juicio hasta que el presidente comience a tomar decisiones controvertidas. Una vez que esto sucede, la cobertura de los medios se vuelve más crítica y el apoyo de la sociedad disminuye (citado por Gronke y Newman, 2003, p. 504). 
En segundo lugar, se considera que la valoración presidencial puede estar condicionada por cuestiones ideológicas o por identificación partidaria: cuando el ciudadano comparte concepciones ideológicas similares al mandatario o se siente identificado con el partido gobernante tiene más probabilidades de aprobar la gestión. El nivel de impacto de la identificación partidaria sobre la popularidad presidencial depende a su vez de la polarización existente en la sociedad (en contextos de mayor polarización ideológica, el impacto es mayor que en circunstancias de menor polarización) y del nivel educativo. Esta misma corriente de pensamiento se aplica a la hora de analizar las razones de voto.

Paul Sniderman (1991) profundizó sobre el condicionamiento de la ideología en el voto a partir de la siguiente premisa: los términos liberalismo y conservadurismo son complejos para ser definidos por el ciudadano medio de Estados Unidos. Sin embargo, muchos de ellos son capaces de definirse ideológicamente. Esta dicotomía se resuelve según el grado de arraigo o intensidad de la ideología con la que se define la persona. Este razonamiento permitiría comprender la presunta inconsistencia entre la autodefinición ideológica abstracta (como liberalismo y conservadurismo) y las opiniones en temas concretos. En cuanto al impacto diferencial de la ideología a partir del nivel educativo, Sniderman concluye que el nivel de información de que disponen los ciudadanos diferencia el proceso de decisión del voto. Mientras que aquellos interesados en política o con mayor nivel de información pueden comparar al presidente actual con los opositores, los ciudadanos con menor nivel de información no pueden realizar esta diferenciación.

Por su parte, William Jacoby (2004) argumenta que en las elecciones presidenciales de Estados Unidos de 2000 la ideología no influyó de manera directa en el voto. Pero sí lo hizo de manera indirecta, es decir, condicionó las opiniones sobre aquellas variables que ejercían un impacto directo sobre la decisión del sufragio.

En cuanto a la simpatía o afinidad partidaria, Martin Watternberg (1991) describe el surgimiento de una nueva era política (surgida en la década del 80 en Estados Unidos y extendida al resto de los países), cuyo centro es el candidato y ya no el partido político. Así es como se desarrolló un proceso de declinación de las identidades partidarias, dando lugar a un proceso de despartidización y mayor preponderancia del elector independiente.

En Argentina, desde el retorno de la democracia, las lealtades partidarias fueron sucumbiendo generando un proceso de "despartidización", ejemplificado en el siguiente gráfico: 
Gráfico 1. ¿Está usted afiliado, simpatiza, no simpatiza pero podría llegar a simpatizar o nunca va a sentir simpatía por ningún partido político?

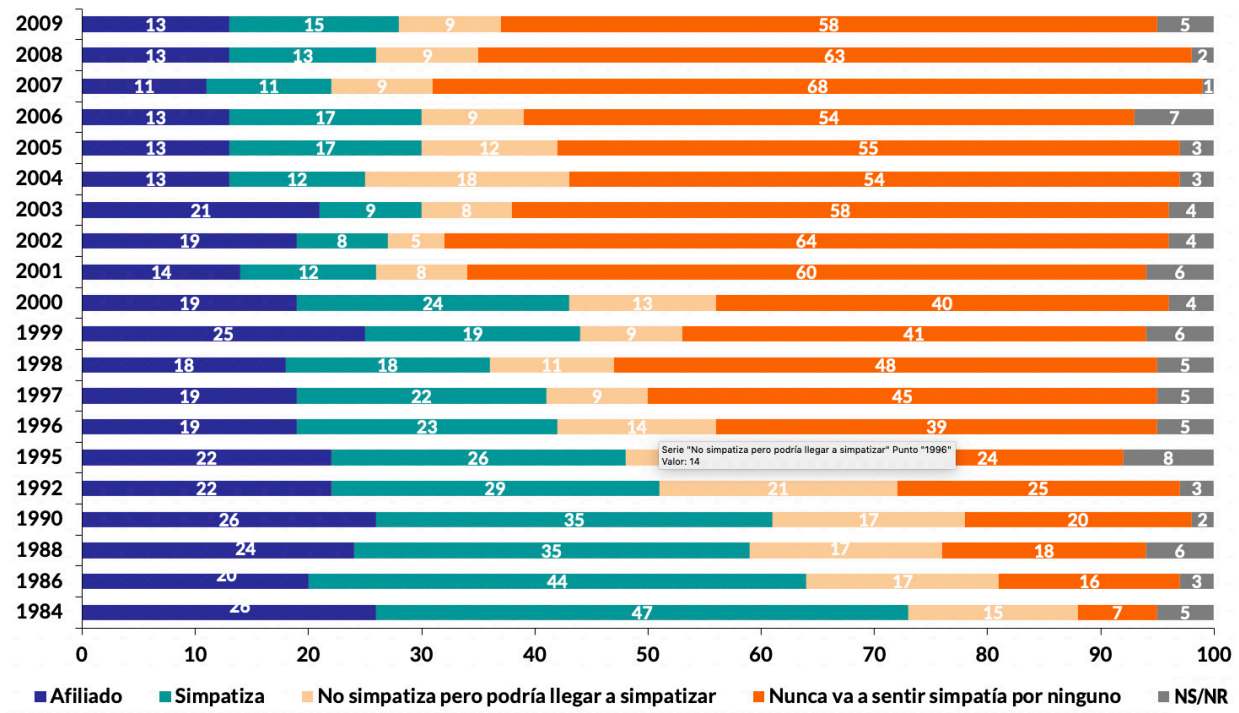

Encuesta en principales ciudades del país. Universo: hombres y mujeres de 18 a 70 años de todos los niveles sociales. Entre 800 y 1200 casos, según año.

Fuente: Ipsos Mora y Araujo.

En tercer lugar, el factor económico es un elemento que impulsa la popularidad presidencial. Es decir, el ciudadano realiza un análisis respecto a la situación económica y premia o castiga al gobernante según los logros obtenidos. Dicho análisis puede sustentarse en lo realizado por el gobierno (mirada retrospectiva) o en las expectativas futuras (mirada prospectiva).

A su vez, el condicionante económico puede fundamentarse en la situación personal del individuo (eje egotrópico) o en el estado de la sociedad en su conjunto (eje sociotrópico).

Robert Erikson, Michael MacKuen, y James Stimson (1992) grafican estos debates en la disyuntiva de campesinos o banqueros. Como campesinos, los ciudadanos evalúan a los líderes mirando hacia atrás de acuerdo a su experiencia económica personal. Como banqueros, los ciudadanos condicionan su evaluación previendo el futuro de la economía del país en su conjunto. Finalmente, los autores concluyen que, al momento de evaluar a un presidente, los ciudadanos actúan como banqueros (citado por Erikson, MacKuen y Stimson, 2000, p. 296). El interrogante que surge es cómo podría el electorado premiar o castigar a un gobierno cuando la información de que dispone es escasa. La explicación a esta 
disyuntiva se basa en que los ciudadanos están expuestos a gran cantidad de información sobre la economía y, además, para la construcción de expectativas sobre el futuro se considera la información adquirida y el conocimiento del pasado reciente. Erikson et al. afirman que "cuando postulamos que las personas actúan racionalmente, lo decimos en el sentido de la frase de Popkin 'racionalidad de información limitada'. La mayoría de las personas no son economistas ni financieros y no exploran profundamente la naturaleza de la economía. Sin embargo, las personas son productores y consumidores cotidianos y, por lo tanto, participan en el aprendizaje durante el curso de sus vidas económicas" (Erikson et al., 2000, p. 296).

Algunas investigaciones demostraron que el impacto de la economía sobre la aprobación presidencial depende de dos factores: el contexto y la institucionalidad. En cuanto al primero, Stokes (2001) afirma que, en momentos de cambio económico o político, el condicionante económico se desactiva: los ciudadanos pueden apoyar al Ejecutivo aun en un contexto de tensión económica (citado por Ortiz Ayala y García Sánchez, 2014, p. 376). En cuanto al factor institucional, la existencia de un factor económico como condicionante de la popularidad presidencial depende de la identificación clara de que el Ejecutivo es el responsable del manejo de la economía (citado por Ortiz Ayala y García Sánchez, 2014, p. 376). En este aspecto, el tipo de gobierno será aquello que medie entre ambos fenómenos.

Teniendo como sustento la teoría del voto económico, Helmut Norpoth (2004) se preguntó cómo fue posible que el Partido Demócrata no pudiera retener la presidencia en las elecciones de Estados Unidos del año 2000 si todos los indicadores económicos eran positivos. Según esta corriente, el voto retrospectivo debió haber beneficiado a Al Gore dada la bonanza económica de la gestión de Clinton.

El autor realizó tres consideraciones respecto al voto retrospectivo:

1. El voto económico es asimétrico: Buenos momentos económicos otorgan menos beneficios electorales para el partido gobernante que los costos en caso de malos momentos económicos. Incluso, este fenómeno es mayor luego de un período de bonanza de varios años, en los cuales el votante se acostumbra a él.

2. "Los electores son banqueros": Lo importante es el futuro y no el pasado.

3. El voto económico requiere una configuración institucional especial: Actúa de manera fuerte cuando el presidente se presenta nuevamente en las elecciones y no ejerce su influencia si el candidato es otro. Es decir, el logro de la bonanza económica corresponde a una persona y el crédito no se transfiere fácilmente.

Complementando este último punto, Lewis-Beck (2014) demostró que, en contextos económicos complejos, el impacto negativo de la economía en la aprobación 
presidencial fue aproximadamente el doble que el impacto positivo en contextos de bonanza económica (citado por Pedro Magalhães, 2020, p. 6).

Un cuarto fundamento asocia las percepciones del electorado sobre la personalidad del funcionario y su evaluación. Así es que, si se considera que el mandatario reúne las condiciones para liderar un país, habrá mayor propensión a aprobarlo. En este caso, atributos como carisma, cercanía, liderazgo son valorados por la población. En consecuencia, los presidentes personalistas tienden a tener mayores niveles de popularidad que presidentes no personalistas (Ortiz Ayala y García Sánchez, 2014). Los autores entienden los gobiernos personalistas como una estrategia que permite mantener una relación directa, afectiva y emocional con sus ciudadanos. Es este vínculo afectivo el que genera una confianza especial con el magistrado, lo cual redunda en mayores niveles de aprobación. Además, cuando la economía no camina por senderos virtuosos la sociedad tiende a castigar en menor medida al funcionario que establece este tipo de vinculación con la ciudadanía.

A su vez, James Beck, Alison Carr y Philip Walmsley (2012) argumentan que los líderes con carisma tienden a tener niveles de aprobación más elevados y a moderar sus caídas en comparación con aquellos funcionarios que carecen de esta característica personal. Finalmente, concluyen asumiendo que las consecuencias de una crisis en la aprobación presidencial dependen del nivel de carisma con el cual responde a ese contexto complejo. Presidentes carismáticos pueden transformar las crisis en oportunidades para obtener apoyo público. Por el contrario, las crisis pueden profundizar sus efectos negativos para los presidentes menos carismáticos.

Por último, en momentos de fuerte crisis e incertidumbre puede generarse un clima de patriotismo por medio del cual la sociedad se encolumna detrás del presidente para superar la crítica realidad. Este fenómeno se conoce como to rally around the flag, es decir, los ciudadanos se unen detrás de la patria y el gobernante para, juntos, sobreponerse a los obstáculos del momento (Sosa Villagarcía y Hurtado Lozada, 2021)

Mueller (1970) fijó tres características que debía reunir un acontecimiento para generar el efecto señalado: a) ser un episodio internacional, b) involucrar a todo el país (Estados Unidos) y especialmente al presidente y c) ser específico y dramático (citado por Newman y Forcehimes, 2010, p. 146). A partir de este estudio inicial sobre el fenómeno to rally around the flag, diversas investigaciones sugirieron algunas modificaciones en la clasificación de los eventos capaces de generar este incremento de la popularidad presidencial. Kernell (1978) señaló que un episodio capaz de generar un crecimiento abrupto de la aprobación presidencial debe tener presencia significativa en las portadas de los diarios más importantes. A su vez, Ostrom y Simon (1985) y Ostrom y Smith (1992) fijaron seis tipos de eventos que afectan la valoración pública de un presidente: crisis internacionales, logros diplomáticos, acontecimientos que impactan directamente en la personalidad del funcionario (especialmente casos de salud), escándalos, disturbios internos y policy 
initiatives (citados por Newman y Forcehimes, 2010, p. 146). Los primeros tres afectan de manera positiva la aprobación del presidente y los últimos tres lo hacen de manera negativa.

Por otro lado, la interpretación de cuál es el motivo por el cual ante estos eventos se produce un busco crecimiento de la popularidad presidencial se dividió en dos corrientes. Una de ellas considera que ante crisis internacionales el encolumnamiento detrás de la figura del presidente se da porque es un símbolo de la unidad nacional o del patriotismo. Una segunda vertiente señala que en tales momentos de crisis la oposición tiende a abstenerse de comentar el desempeño del gobierno nacional y, por lo tanto, escasean noticias críticas hacia la labor del presidente.

A modo de ejemplo, M. Hetherington y M. Nelson (2003) ubican lo sucedido con la aprobación del presidente de Estados Unidos George Bush luego del ataque del 11 de septiembre de 2001 como un fenómeno de estas características. Según Gallup, la evaluación positiva de su administración el 10 de septiembre era del $51 \%$ y a los cinco días posteriores trepó al $86 \%$. Este salto significativo y repentino de su popularidad marcó el mayor crecimiento de una medición a otra, marcó un récord en las series históricas de medición y, por último, el fenómeno to rally around the flag fue el más prolongado en la historia norteamericana. La unidad nacional también se observó en la evaluación que hacían de Bush según la identificación partidaria. Según una encuesta de Gallup, un mes antes de los ataques a las Torres Gemelas, el $89 \%$ de los republicanos aprobaban al presidente, contra un $53 \%$ de los independientes y un $28 \%$ de los demócratas. Diez días posteriores a la tragedia, el $98 \%$ de los republicanos, el $91 \%$ de los independientes $(+38)$ y el $84 \%$ de los demócratas $(+56)$ valoraban al funcionario.

\section{PREGUNTAS A INVESTIGAR Y BASE DE DATOS}

La propagación del coronavirus generó consecuencias económicas (caída del producto, menor consumo, disminución de la recaudación, entre otros), sanitarias (preocupación por la capacidad del sistema para atender a todos los contagiados) y psicológicas (fruto del aislamiento obligatorio impuesto). Y Alberto Fernández encontró en este nuevo contexto la oportunidad de reafirmar su autoridad. Este objetivo, como se dijo, requiere de un elemento fundamental: el apoyo de la ciudadanía. Considerando estas cuestiones, en la presente investigación se intentará dar respuestas a las siguientes preguntas:

1. ¿Cuál es el impacto de la pandemia sobre la figura del presidente y sobre la consolidación de su autoridad?

2. ¿Cuáles son los motivos que condicionan la aprobación del presidente antes y durante la pandemia del coronavirus? 
El análisis abarcará los meses de febrero, marzo y abril. Es decir, el mes previo al primer contagio de COVID-19 en el país y los siguientes dos².

El primer eje de investigación brindará herramientas para evaluar la posible consolidación del liderazgo presidencial como consecuencia del COVID-19 a partir de un crecimiento de su popularidad. Las respuestas a la segunda pregunta podrán dar una idea o abrir el interrogante respecto a la perdurabilidad de los efectos sobre la figura de Alberto Fernández: si la pandemia se transforma en el elemento central que impulsa la aprobación presidencial (y, por ende, se produce una consolidación de su liderazgo), ¿qué pasará en la etapa postcoronavirus?

Para abordar estas temáticas, se utilizaron datos suministrados por Poliarquía Consultores. La primera pregunta de la investigación intentará ser analizada a través de un monitoreo diario que realizó la consultora desde el 13 de marzo hasta el 30 de abril por medio de encuestas por IVR. Se trató de una encuesta nacional donde participaron mayores de 18 años residentes de ciudades de más de 10000 habitantes.

Para la segunda temática de la investigación, se utilizaron encuestas realizadas de manera online a un panel propiedad de la consultora. El mismo tiene la particularidad de que sus miembros fueron seleccionados de manera aleatoria y probabilística. Para identificar las variables que condicionaban la aprobación presidencial antes de la instauración del aislamiento social y obligatorio en el país se empleó una encuesta realizada entre el 4 y el 13 de febrero, con un total de 1000 casos. Para la comparación producto del impacto del coronavirus, se utilizó una encuesta que se realizó entre el 10 y el 14 de abril a un total de 988 miembros.

\section{IMPACTO DEL CORONAVIRUS SOBRE LA FIGURA DEL PRESIDENTE}

El 3 de marzo se detectó el primer caso de COVID-19 en el país y diez días después comenzó el monitoreo de una serie de indicadores que reflejan el estado de la opinión pública frente a la pandemia.

El primer indicador monitoreado fue el nivel de preocupación por el avance del coronavirus en el país. El 13 de marzo, fecha en la cual Argentina contabilizó 34 casos confirmados (Ministerio de Salud de la Nación, 2020), el 48 \% de los encuestados señalaba estar muy preocupado por la pandemia y el $34 \%$ bastante preocupado. La categoría "muy preocupado" fue aumentando progresivamente hasta llegar a su valor más alto el 23 de marzo: $77 \%$. En apenas diez días, el nivel de preocupación creció algo más del 50 \%. Este pico se registra luego del establecimiento del aislamiento social, preventivo y obligatorio en Argentina. A partir de aquella

2. Lo acontecido en el país a partir del mes de mayo queda excluido de la investigación. 
fecha, comienza un proceso de descenso hasta posicionarse, a fines de abril, en valores similares a los del inicio de la serie.

\section{Gráfico 2. ¿Cuánto le preocupa el avance del coronavirus?}

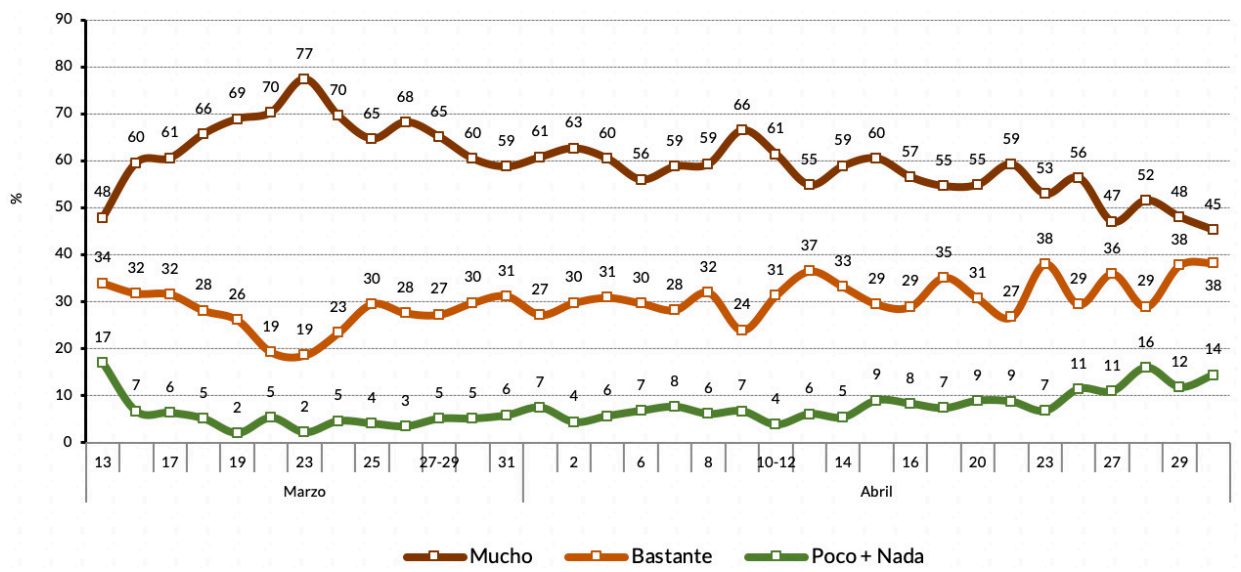

Fuente: Poliarquía Consultores, 2020.

A su vez, Alberto Fernández tomó debida cuenta de este nuevo contexto, asumió el liderazgo en esta "guerra", como la definieron algunos, y transformó la crisis del coronavirus en una oportunidad política. Pasó de decir que "No deberíamos alarmarnos" (ante el primer caso detectado en el país) a "Estamos peleando contra un ejército invisible". Pasó de estar en segundo plano cuando se comunicaban las novedades de la epidemia en el país a comunicar personalmente las acciones que se tomaron para combatir el avance del coronavirus.

Este giro lo llevó, el 15 de marzo, a dictar las primeras medidas (suspensión de clases, licenciamiento de los mayores de 60 años y cierre de fronteras) y el 19 a establecer el aislamiento social, preventivo y obligatorio en el país. En las primeras dos conferencias de prensa que realizó para anunciar las medidas contra la pandemia dejó en claro su rol de líder ("Les aseguro que me voy a poner al frente para tratar de evitar que el ritmo del contagio se acelere"), su autoridad y firmeza ("En esto voy a ser inflexible: los que tengan que hacer la cuarentena van a cumplir la cuarentena y si no la cumplen los perseguiremos penalmente"), su actitud no alarmista pero sí previsora ("Estas medidas no suponen ningún agravamiento de la situación que teníamos, simplemente queremos evitar el crecimiento exponencial de los contagiados"), su vocación de diálogo ("Con el gobernador de la provincia de Buenos Aires y el jefe de Gobierno de la Ciudad venimos trabajando codo a codo desde el primer día") y su búsqueda racional por explicar las medidas ("Hemos cerrado las fronteras de Argentina [...] ¿Por qué lo hacemos? Porque el 
episodio del coronavirus no viene solamente de Europa, está empezando a afectar a países limítrofes").

Según los datos relevados, el nivel de aprobación presidencial creció de manera constante hasta fin de marzo. Alberto Fernández comenzó a transitar el problema del coronavirus en el país con un 43 \% de aprobación y fue ascendiendo hasta su valor más alto (78 \%) a fines de mes. La serie se interrumpe porque la consultora reemplazó esta pregunta por el nivel de apoyo del presidente en relación a su accionar frente a la pandemia y se retoma el 28 de abril. Lo que se observa es, de un mes a otro, una caída de 19 puntos en popularidad que, de todas maneras, significa casi 12 puntos por encima de lo que registraba al inicio de la pandemia.

\section{Gráfico 3. ¿Usted aprueba o desaprueba la gestión de Alberto Fernández como} presidente de la Nación?

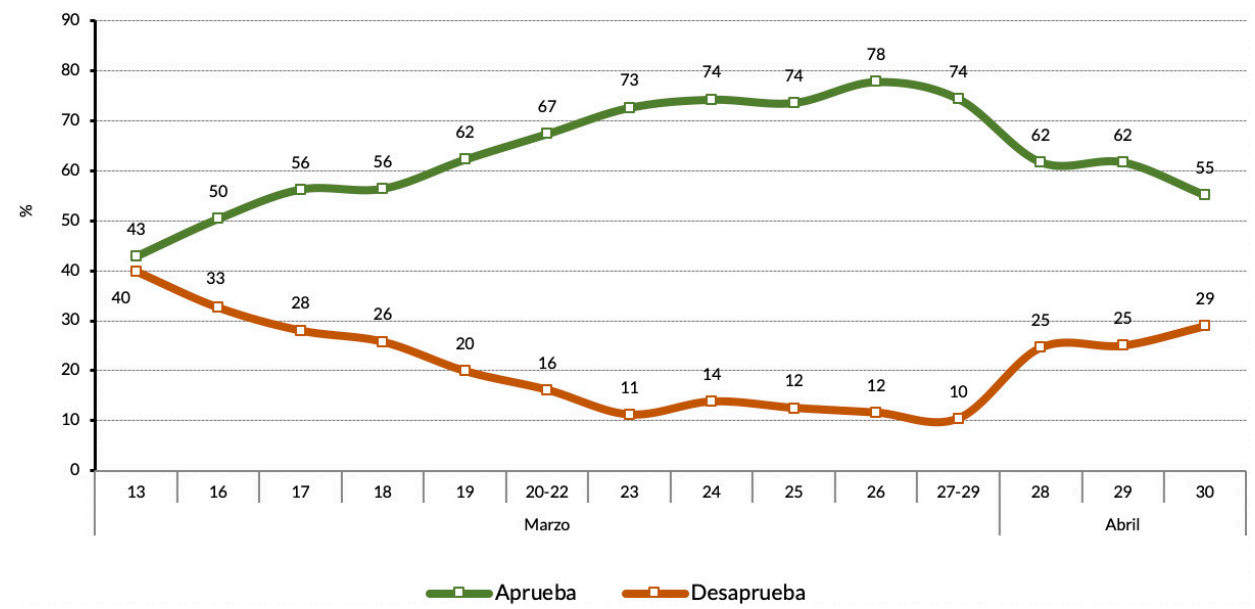

Fuente: Poliarquía Consultores.

¿Por qué razón el presidente tiene un crecimiento de su popularidad de casi 30 puntos en menos de un mes? ¿Y por qué motivos desciende? Los resultados de las encuestas parecieran demostrar que, en situaciones de crisis producto de un enemigo común, cuando la sociedad (incluso el mundo entero) vive preocupada, con temor y angustiada por la realidad que toca enfrentar e incluso cuando hay incertidumbre sobre el futuro, se crea un clima de unión nacional o patriotismo que impulsa a los ciudadanos a encolumnarse detrás del presidente. Se presume la necesidad de confiar en las autoridades en un contexto donde la sociedad está amenazada y requiere volver a la normalidad.

Este fenómeno se observa en el siguiente gráfico, que combina la aprobación presidencial y el porcentaje de encuestados que señalan que les preocupa mucho el 
avance del coronavirus. El nivel de popularidad de Alberto Fernández fue creciendo en paralelo con el aumento de la preocupación por la pandemia. Y, posteriormente, a medida que los argentinos comienzan a acostumbrarse a "convivir con el COVID-19", el temor disminuye de la misma manera que la aprobación. Este efecto conocido como rally around the flag impulsa el crecimiento de la figura del presidente, pero pareciera comenzar a evaporarse.

\section{Gráfico 4. Aprobación presidencial y preocupa mucho el avance del coronavirus}

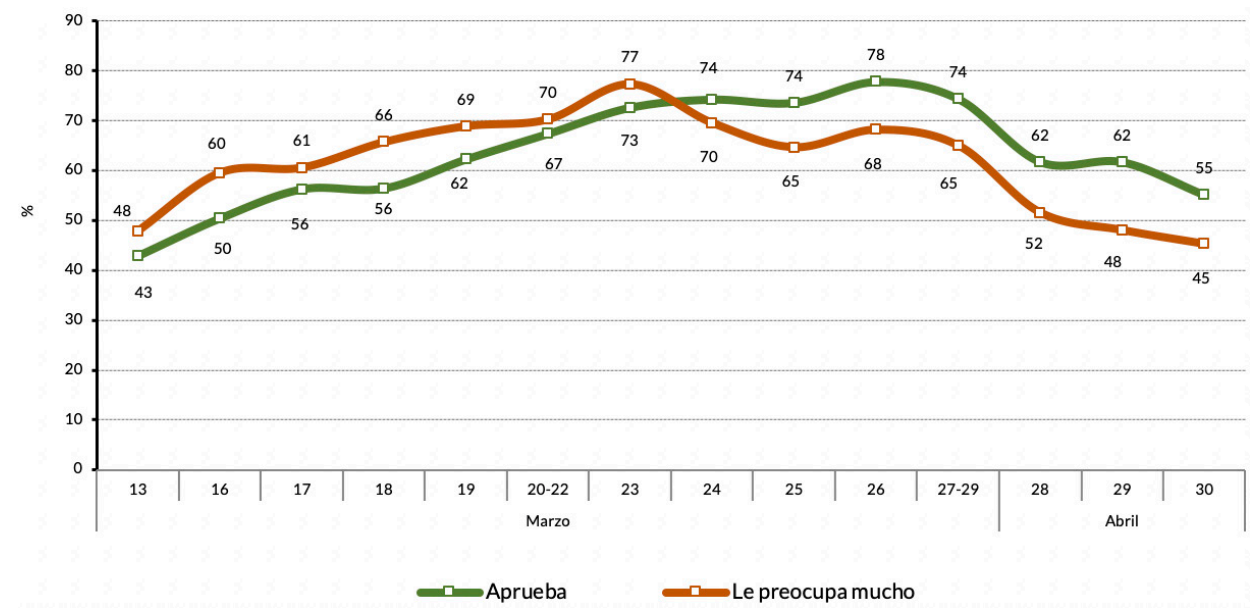

Fuente: Poliarquía Consultores.

Otro signo del auge de cierta unidad nacional como consecuencia de los acontecimientos de la pandemia es la reducción de la lógica de lo que en el país se define como la grieta. La grieta es un término usado en Argentina para marcar la división política que existe entre quienes se reconocen o simpatizan por el kirchnerismo y quienes lo rechazan abiertamente (representados en el partido del expresidente Mauricio Macri). Ambos extremos tienden a rechazar la mayoría de lo que dice o hace el otro. A esta separación sin vasos comunicantes se la denomina grieta.

Una manera de poder identificar estos segmentos es a través de las valoraciones públicas que hacen los ciudadanos de los representantes de cada espectro: Cristina Kirchner y Mauricio Macri. Es así como quienes tienen una imagen positiva de uno y negativa del otro forman parte de un segmento. $Y$ a la inversa, pertenecen al otro extremo ideológico. En el medio, se encuentran los denominados "excluidos" de la grieta.

Según las encuestas telefónicas nacionales de Poliarquía, en julio del año pasado, un mes previo a las elecciones PASO -etapa de mayor ebullición políticael 34 \% se identificaba con el kirchnerismo (es decir, simpatizaban con Cristina 
Kirchner y rechazaban al expresidente), el $26 \%$ con Juntos por el Cambio o el macrismo (apoyaban a Macri y eran detractores de la actual vicepresidenta) y el restante $40 \%$ no se identificaba con ningún extremo del espectro. En abril de este año, en pleno aislamiento social preventivo, los extremos se reducen y crecen los independientes. Quienes no forman parte de la grieta ascienden al $54 \%$ (+14 puntos), aquellos identificados con el kirchnerismo representan el $28 \%$ (-6 puntos) y con Juntos por el Cambio el 18 \% (-8 puntos).

\section{Gráfico 5. Segmentación política}

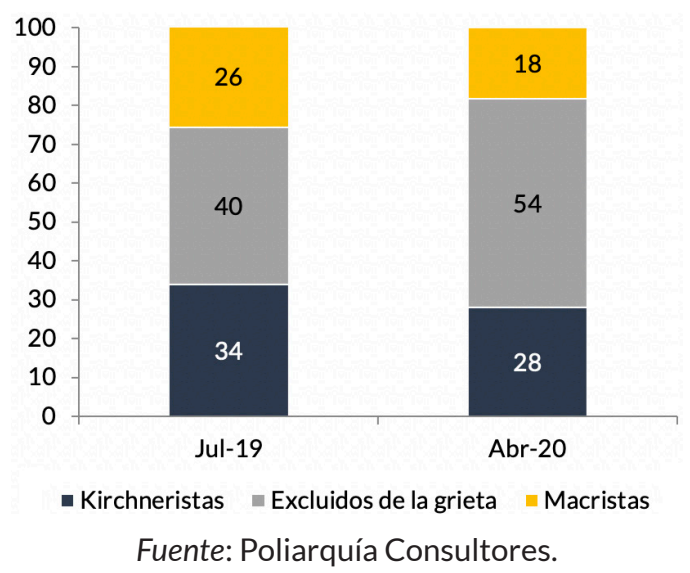

Se trata de dos momentos muy diferentes. En el primero, la lucha agonal por el poder incentivó la división partidaria de los argentinos, registrando que el $60 \%$ de los encuestados se identificara con uno de los dos sectores preponderantes de la política. Casi un año después, en medio de una crisis mundial y con un fuerte retroceso de la actividad económica, ese valor se reduce al 46 \%: la sociedad pareciera exigir dejar a un lado las diferencias y luchas partidarias para, mancomunadamente, buscar soluciones a este fenómeno nunca antes visto. En este cambio en la configuración del electorado parece encontrarse la necesidad de dejar de lado la lucha por el poder para encolumnarse detrás del presidente en esta situación de excepcionalidad.

Durante el período que toma como referencia esta investigación, la consecuencia de estos fenómenos es la consolidación del liderazgo de Alberto Fernández. Así se observa en la tabla siguiente: en marzo solo el $14 \%$ consideraba que el presidente era quien retenía el poder y Cristina Kirchner acataba sus decisiones. Este valor, durante la pandemia, más que se duplicó: pasó a ser el $35 \%$. A su vez, de un mes a otro no solo se redujo drásticamente la idea de que ambos funcionarios compartirían el poder (del $32 \%$ al $9 \%$ ), sino que creció la hipótesis de conflicto entre ellos (del $22 \%$ al $29 \%$ ) a partir del crecimiento de la figura de Alberto Fernández. 
Tabla 2. Según su opinión, ¿cuál de las siguientes opciones refleja mejor la relación que tendrán Alberto Fernández y Cristina Kirchner?

\begin{tabular}{lcc}
\hline & Marzo & Abril \\
\hline $\begin{array}{l}\text { Alberto Fernández será quien tenga el poder y Cristina Kirchner acatará } \\
\text { sus decisiones }\end{array}$ & 14 & 35 \\
$\begin{array}{l}\text { Alberto Fernández y Cristina Kirchner compartirán el poder en una } \\
\text { convivencia pacífica }\end{array}$ & 32 & 9 \\
$\begin{array}{l}\text { Se desatará una lucha de poder entre Alberto Fernández y Cristina } \\
\text { Kirchner }\end{array}$ & 22 & 29 \\
$\begin{array}{l}\text { Cristina Kirchner será quien tenga el poder y Alberto Fernández acatará } \\
\text { sus decisiones }\end{array}$ & 26 & 22 \\
NS/NC & 6 & 4 \\
Total & 100 & 100 \\
\hline
\end{tabular}

Fuente: Poliarquía Consultores, 2020.

Luego de los primeros dos meses del primer caso de coronavirus en Argentina se detectaron los siguientes fenómenos políticos: 1. crecimiento de la preocupación de la opinión pública frente a la pandemia hasta la decisión del gobierno de establecer el aislamiento preventivo, social y obligatorio. 2. Desde esa fecha, descenso de la preocupación ciudadana en sintonía con una evolución de contagios en el país que no superaban los 200 nuevos casos diarios, situación muy diferente a otros países del continente. 3. Crecimiento de la popularidad presidencial en paralelo con el incremento del temor de los argentinos. 4. Ante una disminución de la preocupación social por el coronavirus, se produjo un descenso de la aprobación del presidente. 5. Por último, una reducción de la lógica de la grieta.

\section{FACTORES QUE CONDICIONAN LA APROBACIÓN PRESIDENCIAL}

Por medio de regresiones logísticas se identificarán los factores que condicionan la aprobación del presidente Fernández en dos momentos diferentes: uno en el mes de febrero (cuando el país no había registrado casos de coronavirus) y otro en el mes de abril luego del establecimiento del aislamiento social, preventivo y obligatorio.

En ambos casos, la variable dependiente es aprobación de la gestión (1 = aprueba y $0=$ resto). Las variables independientes son las siguientes: 
A. Evaluación de la situación actual del país: positivo $=1$, regular $=0.5$ y negativo $=0$. El NS/NR fue reemplazado por la media.

A. Evaluación del país con respecto a un año atrás (evaluación retrospectiva): Mejoró $=1$, se mantiene igual $=0.5$ y empeoró $=0$. El NS/NR fue reemplazado por la media.

B. Expectativas de cómo estará el país el próximo año: Mejor $=1$, igual $=0.5$ y peor $=0$. EI NS/NR. fue reemplazado por la media (evaluación prospectiva).

A su vez, se incluyeron por separado dos variables independientes: voto a presidente 2019 y ubicación ideológica. Ambas variables fueron consideradas categóricas en los modelos ${ }^{3}$. Aquella referente a la ideología agrupa a los encuestados en kirchneristas, macristas y los llamados excluidos de la grieta, es decir, ciudadanos que no se identifican con ninguno de los principales espacios del espectro político.

Según la encuesta de Poliarquía del mes de febrero, los resultados son los siguientes:

Tabla 3. Resultados de la regresión logística del mes de febrero ${ }^{4}$

\begin{tabular}{lccc}
\hline & \multicolumn{2}{c}{ Modelo I } & Modelo II \\
\cline { 2 - 4 } & Beta & Probabilidad predicha & Beta \\
\hline Evaluación de la situación actual & $1.905^{* * *}$ & $16 \%$ & $1.695^{* * *}$ \\
Evaluación retrospectiva & $2.125^{* * *}$ & $19 \%$ & $2.246^{* * *}$ \\
Evaluación prospectiva & $3.035^{* * *}$ & $37 \%$ & $2.973^{* * *}$ \\
Voto anterior & & & -- \\
Votantes de Fernández & $3.208^{* * *}$ & $32 \%$ & -- \\
Votantes de otras opciones & $0.783^{*}$ & $15 \%$ & -- \\
Ideología & -- & -- & $3.997^{* * *}$ \\
Kirchneristas & -- & -- &
\end{tabular}

3. La opción de respuesta contra la cual se compararon las variaciones de probabilidad de la ocurrencia del fenómeno estudiado es votantes de Juntos por el Cambio (variable voto anterior) e identificación con el macrismo (variable ubicación ideológica).

4. La columna Beta reporta los coeficientes y errores estándares. La columna Probabilidad reporta el cálculo del cambio en las probabilidades condicionales de aprobar la gestión del presidente al pasar del mínimo al máximo valor de la variable independiente especificada y dejando el resto de variables fijas en sus medias. 


\begin{tabular}{lccc}
\hline & & Modelo I & Modelo II \\
\cline { 2 - 4 } & Beta & Probabilidad predicha & Beta \\
\hline Excluidos de la grieta & -- & -- & $1.775^{* * *}$ \\
Constante & $-2.657^{* * *}$ & $-5.908^{* * *}$ \\
\hline $\mathrm{R}^{2}$ de Nagelkerke & 800 & 761 \\
Porcentaje de acierto del modelo & $90.50 \%$ & $89.80 \%$ \\
Número de observaciones & 819 & 833 \\
\hline
\end{tabular}

Fuente: Elaboración propia a partir de datos de Poliarquía Consultores.

Ambos modelos tienen una capacidad explicativa de la variable dependiente muy similar. En ambos modelos, todas las variables incorporadas son relevantes estadísticamente para explicar la aprobación de Alberto Fernández. En segundo lugar, tienen correlación positiva con la variable dependiente.

En cuanto al primer modelo, que incluye el voto a presidente del año 2019, las dos variables que muestran un mayor efecto son la evaluación prospectiva y el voto en la elección de 2019. Así, pasar de considerar que el próximo año el país estará peor a creer que estará mejor (dejando el resto de las variables fijas en sus medias) aumenta un $37 \%$ la probabilidad de evaluar positivamente el desempeño del presidente. A su vez, pasar de haber votado a Juntos por el Cambio a haber optado por el Frente de Todos incrementa la probabilidad de aprobar a Alberto Fernández en un $32 \%$. También se produce un aumento en la probabilidad de valorar la tarea del presidente para los votantes de otras alternativas (en comparación con los votantes de Macri), aunque en menor medida.

Por último, con incrementos similares en la probabilidad de apoyar al gobierno nacional, se encuentran las variables correspondientes a la evaluación retrospectiva (un $19 \%$ más de probabilidad al pasar de considerar que el país empeoró a creer que mejoró) y a la evaluación de la situación actual (se incrementa la probabilidad en un $16 \%$ ).

Es entendible que la evaluación prospectiva condicione más que la retrospectiva o la evaluación presente dado que el gobierno había asumido hacía apenas dos meses y se generaron expectativas con el cambio de gobierno.

El segundo modelo, que incorpora la variable ubicación ideológica, refleja una preponderancia en el condicionamiento de la ideología. Pasar de considerarse macrista a estar identificado con el kirchnerismo aumenta un $73 \%$ las probabilidades de aprobar al presidente. En tanto, pasar de sentirse simpatizante de Juntos por el Cambio a considerarse independiente incrementa las probabilidades de apoyo a Fernández en un $22 \%$. 
Estos resultados permiten concluir que existe un mayor condicionamiento de la variable ideológica que del voto anterior. La interpretación de este incremento diferencial en las probabilidades se basa en que posiblemente quienes hayan votado al actual presidente no pertenezcan a su núcleo de mayor cercanía y hayan optado por esa opción para evitar un triunfo del por entonces candidato oficialista. Por lo tanto, son menos propensos a aprobar la gestión presidencial que los segmentos que sí se reconocen kirchneristas.

En segundo lugar, este segundo modelo reproduce el mayor condicionamiento del futuro que el pasado o el presente. Si bien en los tres casos pasar de los valores mínimos a los valores máximos de las variables independientes incrementa la probabilidad de aprobar al gobierno del Frente de Todos (incluso en mayor medida que en el primer modelo), las expectativas tienen una influencia mayor: quienes creen que el país estará mejor el año próximo tienen casi un 50 \% más de probabilidad de evaluar positivamente la gestión presidencial que aquellos que creen que el país estará peor.

En abril, luego de un mes de que el coronavirus se instaló en la agenda de los argentinos, el impacto de las variables condicionantes de la aprobación presidencial sufrió modificaciones. En esta oportunidad se incorporó la variable aprobación de gestión de Alberto Fernández en relación al coronavirus ${ }^{5}$ como un indicador indirecto de la preocupación de los ciudadanos por la pandemia. En caso de que esta variable condicione de manera contundente la aprobación del desempeño general de la gestión significa que la preocupación o la relevancia de la problemática es tal para los argentinos que un buen desempeño en esta materia influye de manera positiva en la evaluación general del mandatario. Por el contrario, un control errático de la pandemia podría condicionar en forma negativa la popularidad presidencial. Si no fuese una temática que preocupe a los encuestados, el impacto sobre la valoración presidencial debiera ser bajo o nulo independientemente de cómo los ciudadanos evalúan el combate de la pandemia por parte de la autoridad presidencial. A nivel internacional las aprobaciones presidenciales tuvieron un comportamiento dispar. Por ejemplo, se observaron países que sufrieron consecuencias sanitarias negativas, como Italia (Erlanger, 2020) y, a pesar de ello, la valoración de las autoridades creció. En otros casos, la popularidad del mandatario creció en contextos de acciones positivas para controlar la pandemia (Alemania) y en países cuyos líderes relativizaron la enfermedad hubo fenómenos diversos: decayó la imagen del presidente en Brasil (Pesquisa XP, 2020) y se incrementó en Estados Unidos (Jones, 2020) o se mantuvo elevada en México (Moreno, 2020).

5. ¿Usted aprueba o desaprueba lo que está haciendo Alberto Fernández en relación al coronavirus? (1 = aprueba y 0 = resto). 
Tabla 4. Resultados de la regresión logística del mes de abril (2020)

\begin{tabular}{lccc}
\hline & \multicolumn{2}{c}{ Modelo I } & Modelo II \\
\cline { 2 - 4 } & Beta & Probabilidad predicha & Beta \\
\hline Evaluación de la situación actual & $1.378^{* * *}$ & $4.1 \%$ & $1.103^{* *}$ \\
Evaluación retrospectiva & 451 & $1.5 \%$ & 506 \\
Evaluación prospectiva & $0.810^{* * *}$ & $2.9 \%$ & $0.964^{* * *}$ \\
Voto anterior & & & -- \\
Votantes de Fernández & $2.836^{* * *}$ & $46.6 \%$ & -- \\
Votantes de otras opciones & $1.564^{* * *}$ & $33.8 \%$ & -- \\
Ideología & -- & & $5.634^{* * *}$ \\
Kirchneristas & -- & & $2.007^{* * *}$ \\
Excluidos de la grieta & -- & & $4.503^{* * *}$ \\
Gestión de la crisis del coronavirus & $4.317^{* * *}$ & $69.2 \%$ & $-3.150^{* * *}$ \\
Constante & $-5.11^{* * *}$ & & 617 \\
$\mathrm{R}^{2}$ de Nagelkerke & 599 & & $85.90 \%$ \\
Porcentaje de acierto del modelo & $83.10 \%$ & & 918 \\
Casos incluidos en el análisis & 918 & & \\
\hline
\end{tabular}

${ }^{*} \mathrm{p}<0.1 ;{ }^{* *} \mathrm{p}<0.05 ;{ }^{* * *} \mathrm{p}<0.01$

Fuente: Elaboración propia a partir de datos de Poliarquía Consultores.

El primer modelo, que incorpora el voto anterior, demuestra que la variable que ejerce mayor influencia sobre la aprobación presidencial es la evaluación del trabajo de Alberto Fernández en el combate del coronavirus: pasar de desaprobar su tarea en términos de pandemia a aprobar su labor incrementa las probabilidades de aprobar su desempeño general en un $69 \%$.

La segunda variable que ejerce influencia directa sobre la variable dependiente es el voto: los electores del Frente de Todos y de otras opciones tienen un $47 \%$ y un $34 \%$ más de probabilidad de evaluar positivamente el desempeño del presidente que los votantes de Juntos por el Cambio.

En cuanto al segundo modelo, la identificación ideológica se transforma en la variable más importante a la hora de predecir la aprobación de Alberto Fernández. 
Aquellos que se identifican con el kirchnerismo y aquellos que se reconocen como excluidos de la grieta tienen un $69 \%$ y un $46 \%$ más de probabilidades respectivamente de aprobar el desempeño del funcionario que los ciudadanos cercanos al macrismo.

La segunda variable que condiciona la aprobación general del gobierno es la evaluación del manejo de la pandemia: pasar de desaprobar a aprobar su tarea incrementa las probabilidades de valorar la gestión del mandatario un 50 \%.

En ambos casos se confirma que la pandemia ejerce una influencia preponderante sobre la valoración presidencial. Si el coronavirus no fuera una problemática tal para los encuestados, no debiera impactar en la evaluación de gestión. Y el sentido de la asociación estará dado por cómo los argentinos evalúen el manejo y el control de la pandemia en el país.

En tanto, luego del establecimiento del aislamiento social, preventivo y obligatorio, las variables relacionadas con la evaluación actual del país y con las evaluaciones retrospectiva y prospectiva pasan a tener un condicionamiento marginal (incluso la mirada retrospectiva no es significativa en el segundo modelo). Se trata de una manifestación de que la pandemia modificó las motivaciones para aprobar o desaprobar al presidente.

Tal como ocurrió en el mes de febrero, el impacto de la ideología es mayor que el voto anterior, dado que hay votantes del Frente de Todos que no se identifican con el kirchnerismo, siendo aquel un espacio político que integra a diversos sectores del peronismo y de movimientos sociales.

Por otro lado, otro signo del fenómeno to rally around the flag es el aumento de las probabilidades de aprobar al presidente cuando se trata de ciudadanos que no forman parte de los segmentos más cercanos a él. Previo a la instalación de la cuarentena, quienes no votaron por Fernández tenían un 15 \% más de probabilidades de aprobar su gestión que aquellos que habían votado por Macri. Ahora la probabilidad se incrementa al $34 \%$. En cuanto a los excluidos de la grieta, antes de la cuarentena tenían un $22 \%$ más de probabilidad de aprobar su gestión que aquellos identificados con el macrismo y en abril, la probabilidad es un $46 \%$ mayor que los macristas.

En conclusión, luego de que se instalara la problemática del coronavirus en el país y ganara la notoriedad que tomó, se confirma lo siguiente:

i. La preocupación de la sociedad por el avance de la pandemia se transformó en una variable central que explica los altos niveles de aprobación del presidente.

i. Detrás de esta crisis, los ciudadanos se encolumnaron detrás del mandatario generando una especie de unidad nacional o patriotismo.

ii. Se redujeron las diferencias políticas, logrando que quienes no se sienten cerca del espacio de Alberto Fernández o no lo votaron aumentaran las 
probabilidades de aprobar su gestión luego del primer caso de COVID-19 en el país.

iii. Las variables de evaluación retrospectiva y proyectiva ejercieron influencia condicionante, especialmente, en los primeros meses de su gestión.

\section{CONCLUSIONES}

Alberto Fernández llegó al poder impulsado por la actual vicepresidenta Cristina Kirchner. Esta situación generó dudas en la opinión pública respecto a su poder real, autoridad y liderazgo. En este contexto, la consolidación de su figura era una necesidad central para los primeros meses de su gobierno, para lo cual debía apropiarse de algunos logros de gestión que le permitieran lograr un respaldo contundente de la ciudadanía.

Frente a esta necesidad y ante escasos signos de recuperación económica, el coronavirus estalló en el país. A medida que la enfermedad avanzaba, la sociedad mostraba mayor preocupación por la pandemia. Y en paralelo al aumento del temor ciudadano, los niveles de apoyo al presidente trepaban a valores muy elevados. Con el transcurrir de los días, la preocupación de los argentinos por el avance del coronavirus descendía y, a su vez, la valoración presidencial recorría el mismo camino. Es decir, la preocupación social por el COVID-19 fue un aliado central de Alberto Fernández y que condicionó la aprobación de su gobierno: a mayor preocupación, mayor impacto de la pandemia sobre su popularidad. Y a menor preocupación, menor impacto.

A su vez, además de un encolumnamiento detrás del presidente, la sociedad argentina redujo la lógica de la grieta: más ciudadanos no se reconocían cercanos de los extremos partidarios (kirchneristas o antikirchneristas).

Como consecuencia de estos fenómenos, el presidente comenzó a revertir aquella duda inicial en cuanto a quién toma las decisiones en el gobierno, abriendo un espacio para el reconocimiento del liderazgo de Alberto Fernández.

No obstante, y considerando la influencia determinante de la pandemia sobre la evaluación de gobierno y, por ende, sobre la autoridad del presidente, el interrogante acerca de qué sucederá con su figura una vez que se controle o se profundice la enfermedad o la sociedad se acostumbre a convivir con ella está latente: ¿los niveles de aprobación de Alberto Fernández descenderán? ¿Resurgirán las dudas sobre quién ostenta el poder real en el gobierno?

Con el tiempo se sabrá si la consolidación de su figura fue apenas un oasis en sus cuatro años de gestión o si se trató de una conquista personal. Para esto se deberá tener en cuenta si la sociedad le reconoce que sus medidas (aislamiento social) evitaron una crisis sanitaria como en otros países o si, por el contrario, las 
consecuencias económicas de la cuarentena son tan fuertes que reavivan el debate sobre su gobierno.

\section{BIBLIOGRAFÍA}

Beck, J., Carr, A. y Walmsley, Ph. (2013). Bankers or peasants revisited: economic expectations and presidential approval. Electoral Studies, 23, 934-942.

Cabezas, J. M. y Navia, P. (2019). Presidential approval in Chile, 1990-2018: Variance in UShaped curves. Revista Latinoamericana de Opinión Pública, 8(2).

Carlin, R., Martínez-Gallardo, C., Hartlyn, J., Hellwing, T. y Love, G. (2018). Public Support for Latin American Presidents: The Cyclical Model in Comparative Perspective. Research and Politics, 5(3).

Erikson, R., MacKuen, M. y Stimson, J. (2000). Bankers or peasants revisited: economic expectations and presidential approval. Electoral Studies, 19, 295-312.

Erlanger, S. (15 de abril de 2020). Coronavirus Has Lifted Leaders Everywhere. Don't Expect That to Last. The New York Times. Recuperado de https://www.nytimes. com/2020/04/15/world/europe/coronavirus-presidents.html.

García Sánchez, M. y Rodríguez Raga, J. C. (2019). Personality and an internal enemy: understanding the popularity of Álvaro Uribe, 2002-2010. Revista Latinoamericana de Opinión Pública, 8(2).

Gronke, P. y Newman, B. (2003). FDR to Clinton, Mueller to?: A Field Essay on Presidential Approval. Political Research Quarterly. Recuperado de https://www.researchgate.net/ publication/249802426.

Hetherington, M. y Nelson, M. (2003). Anatomy of a Rally Effect: George W. Bush and the War on Terrorism. Political Science and Politics, 36(1), 37-42.

Jacoby, W. (2004). Ideology in the 2000 Election: A study in ambivalence. En H. Weisberg y C. Wilcox (Eds.), Models of voting in Presidential elections. The 2000 U.S. Election (pp. 103119). California: Stanford University Press.

Jones, J. (24 de marzo de 2020). President Trump's Job Approval Rating Up to 49 \%. Recuperado de https://news.gallup.com/poll/298313/president-trump-job-approval-rating.aspx.

Klobovs, L. (2015). Factores estructurales y no estructurales que influyeron en la decisión del voto en las elecciones presidenciales de 2007 y 2011 (Tesis de maestría). Universidad Nacional de Tres de Febrero. Buenos Aires.

Magalhães, P. (2020). Public Opinion and Executive Approval. The Oxford Handbook of Political Executives.

Maquiavelo, N. (2004). El Príncipe. Alicante: Biblioteca Virtual Miguel de Cervantes. Recuoperado de http://www.cervantesvirtual.com/nd/ark:/59851/bmcxg9z9.

Ministerio de Salud de la Nación. (13 de marzo de 2020). Informes diarios. Recuperado de https://www.argentina.gob.ar/coronavirus/informe-diario?utm_source=search\&utm_ medium $=c p c \& u t m \_c a m p a i g n=$ coronavirus\&utm_term=grants\&utm_content=nacional

Moreno, A. (6 de abril de 2020). Cae aprobación de AMLO a $60 \%$. El Financiero. Recuperado de https://www.elfinanciero.com.mx/nacional/cae-aprobacion-de-amlo-a-60. 
Murillo, V. y Visconti, G. (2017). Economic performance and incumbents' support in Latin America. Electoral Studies, 47, 180-190.

Newman, B. y Forcehimes, A. (2010). "Rally Round the Flag" Events for Presidential Approval Research. Electoral Studies, 29, 144-154.

Norpoth, H. (2004). Bush v. Gore: The recount of Economic voting. En H. Weisberg y C. Wilcox (Eds.), Models of voting in Presidential elections. The 2000 U.S. Election (pp. 49-64). California: Stanford University Press.

Ortiz Ayala, A. y García Sánchez, M. (2014). Porque te quiero te apoyo: Estilo de gobierno y aprobación presidencial en América Latina. Revista de Ciencia Política, 34(2).

Pesquisa XP. (20 de mayo de 2020). Tendência de aumento na reprovação ao presidente Jair Bolsonaro. Recuperado de https://conteudos.xpi.com.br/politica/pesquisa-xp-maio2020-tendencia-de-aumento-na-reprovacao-ao-presidente-jair-bolsonaro/.

Pignataro, A. y Cascante, M. J. (2019). Presidential approval in Costa Rica: Explaining typical and unusal patterns. Revista Latinoamericana de Opinión Pública, 8(2).

Ratto, C. (2020). Otra vez la economía. La influencia de la agenda económica en las elecciones 2019. Más Poder Local, 40, 39-56.

Rivas Otero, J. M. (2012). Liderazgo político y gobernabilidad en América Latina: una aproximación teórica y metodológica. XV Encuentro de Latinoamericanistas Españoles. Madrid. pp. 312-334.

Rodríguez Yerba, M. (13 de junio de 2020). Un peronismo resignado, a los pies de Cristina Kirchner. La Nación. Recuperado de https://www.lanacion.com.ar/politica/ un-peronismo-resignado-a-los-pies-de-cristina-kirchner-nid2378368.

Selios, L. (2019). Aprobación presidencial y claridad de la responsabilidad en un contexto de cambio político e institucional: el caso uruguayo. Revista Latinoamericana de Opinión Pública, 8(2).

Sniderman, P., Brody, R. y Tetlock, P. (1991). Reasoning and choice. Explorations in Political psychology. Cambridge: Cambridge University Press.

Sosa-Villagarcia, P., y Hurtado Lozada, V. (2021). Covid-19 and Presidential Popularity in Latin America. Revista Latinoamericana de Opinión Pública, 10(2). https://doi. org/10.14201/ rlop.23664

Tagina, M. L. (2012). Factores contextuales, predisposiciones de largo plazo y accountability electoral en Argentina en tiempos del Kirchnerismo. Política y Gobierno, 19(2).

Wallace, A. (2020). Coronavirus: por qué la pandemia de covid-19 está aumentando la popularidad de la mayoría de los presidentes. BBC News Mundo. Recuperado de https://www. bbc.com/mundo/noticias-america-latina-52186602.

Wattenberg, M. (1991). The Rise of Candidate-Centered Politics. Presidential Elections of the 1980s. Londres: Harvard University Press. 